\title{
Multiple Dural Arteriovenous Fistulas Presenting as Rapidly Progressive Dementia
}

\author{
Nuno Mendonça, MD, * Gonçalo Santos, MD, $\dagger$ Diana Duro, MSc, * Egídio Machado, MD, $\dagger$ \\ Augusto Goulão, PhD, MD, $\ddagger$ and Isabel Santana, PhD, MD*
}

\begin{abstract}
Introduction: Dural arteriovenous fistulas (DAVFs) are important causes of neurological dysfunction and are many times misdiagnosed. Particularly in older populations, DAVFs may present with a selective cognitive dysfunction.
\end{abstract}

Case Report: The authors describe a 70-year-old woman presenting with a rapidly progressive dementia, very similar in presentation to prion disease. Neuroimaging showed multifocal DAVFs associated with venous thrombosis and white matter changes, suggesting that impaired cerebral circulation due to venous hypertensive encephalopathy caused the patient's dementia. Prompt treatment of some of the abnormal shunts with endovascular embolization resulted in a clinically relevant improvement. Subsequent clinical improvement was achieved with anticoagulation, although no cause or predisposing factor was documented that could have led to the development of the venous thrombosis.

Conclusions: Neurologists should maintain a high degree of suspicion to avoid missing the diagnosis of DAVFs that are potentially treatable lesions.

Key Words: cerebral blood flow, dementia, interventional, sinus thrombosis, thrombophilia

(The Neurologist 2012;18:130-132)

$\mathrm{D}$ ural arteriovenous fistulas (DAVFs) are cerebral vasculopathies that present with great clinical heterogeneity. In most cases, they present with pulsatile tinnitus, headache, or orbital symptoms such as chemosis and proptosis; other much less frequent presentations include dementia, ${ }_{1}^{1}$ parkinsonism, or focal neurological dysfunction. ${ }^{2}$ Clinical reports have also emphasized the importance of early diagnosis and treatment. ${ }^{2}$

Mounting evidence suggests that DAVFs are acquired lesions, although the etiology is still controversial. A tentative theory suggests that DAVFs are neoangiogenesis disorders of dural vessels caused by dural sinus thrombosis, infection, or inflammation. ${ }^{3}$

\section{CLINICAL CASE}

A 70-year-old woman presented with a 2-week history of amnesia, language difficulties, incoherent speech, and progressive gait disturbance. Her past medical history did not reveal any head trauma, skull surgery, meningitis, cancer, or thrombophlebitis. There was no cognitive impairment before symptom onset. A

From the Departments of *Neurology; $†$ Neuroradiology, University Hospital of Coimbra, Coimbra; and †Department of Neuroradiology, Hospital Garcia de Orta, Almada, Portugal.

The authors declare no conflict of interest.

Reprints: Nuno Mendonça, MD, Department of Neurology, University Hospital of Coimbra, Praceta Mota Pinto, 3000-075 Coimbra, Portugal. E-mail: nunomig.mendonca@gmail.com.

Copyright (C 2012 by Lippincott Williams \& Wilkins

ISSN: 1074-7931/12/1803-0130

DOI: $10.1097 /$ NRL.0b013e318251e695 head computed tomography scan showed mild cortical atrophy, and a diagnosis of degenerative dementia was proposed.

The patient's symptoms rapidly progressed, and 3 weeks later, she was admitted to our hospital.

On neurological examination, she was alert but uncooperative, with spontaneous myoclonus of the limbs, occasionally with startle reaction. Besides akinetic mutism and myoclonus, she had a flaccid tetraparesia with brisk tendon reflexes, extensor plantar responses, and exuberant primitive reflexes. There was no papilloedema, head or neck bruits, and during the disease evolution, she never complained of headache or visual impairment.

The strongest clinical suspicion at this point was Creutzfeldt-Jakob disease, and an electroencephalogram showed diffuse slowing of cerebral activity with periods of delta waves bilaterally, predominantly in the right hemisphere. No pattern suggestive of prion disease, like continuous periodic stereotypic sharp waves, was present.

The magnetic resonance imaging showed exuberant dilated veins localized to the cerebellar hemispheres, right cerebral hemisphere (including dilated pial veins), and, in a less marked manner, to the left cerebral hemisphere. Also, there was T2 hyperintensity of the cerebellar white matter, the white matter of the right temporal lobe extending to the external capsule, and the posterior limb of the ipsilateral internal capsule and centrum semiovale (Fig. 1A). None of these white matter changes showed restricted water diffusion on apparent diffusion coefficient maps.

The patient underwent cerebral angiography (Fig. 1B) that showed DAVFs with separate shunts located at the sagittal sinus, the torcula, and the transverse sinuses bilaterally. There was cortical venous reflux and marked venous congestion both in supra and infratentorial regions (type II A + B Cognard classification and type II Borden classification). Associated with the DAVFs were multiple thrombi adjacent to the torcular area, including a superior transverse sinuses thrombosis, leading to venous occlusion/stenosis in both transverse sinuses, with additional occlusion of the left sigmoid sinus. The involved dural sinuses receive arterialized blood flow from the arteriovenous shunts, with turbulent flow within the superior sagittal sinus and retrograde drainage to the deep venous system and cortical veins.

The angiographic diagnostic procedure was followed by endovascular treatment that consisted of transarterial embolization of branches of the middle meningeal and occipital arteries with $N$-butyl-cyanoacrylate, without complications.

After the embolizations, the patient's clinical condition progressively improved. At 1 month, she was cooperative and had insight to her illness, although there remained severe psychomotor slowness. The Portuguese version of the Mini-Mental State Examination (MMSE) ${ }^{4,5}$ score was 15 (compatible with mild dementia). Pretreatment MMSE was impossible to obtain due to the patients severe cognitive impairment. A more comprehensive neuropsychological evaluation was aborted due to 

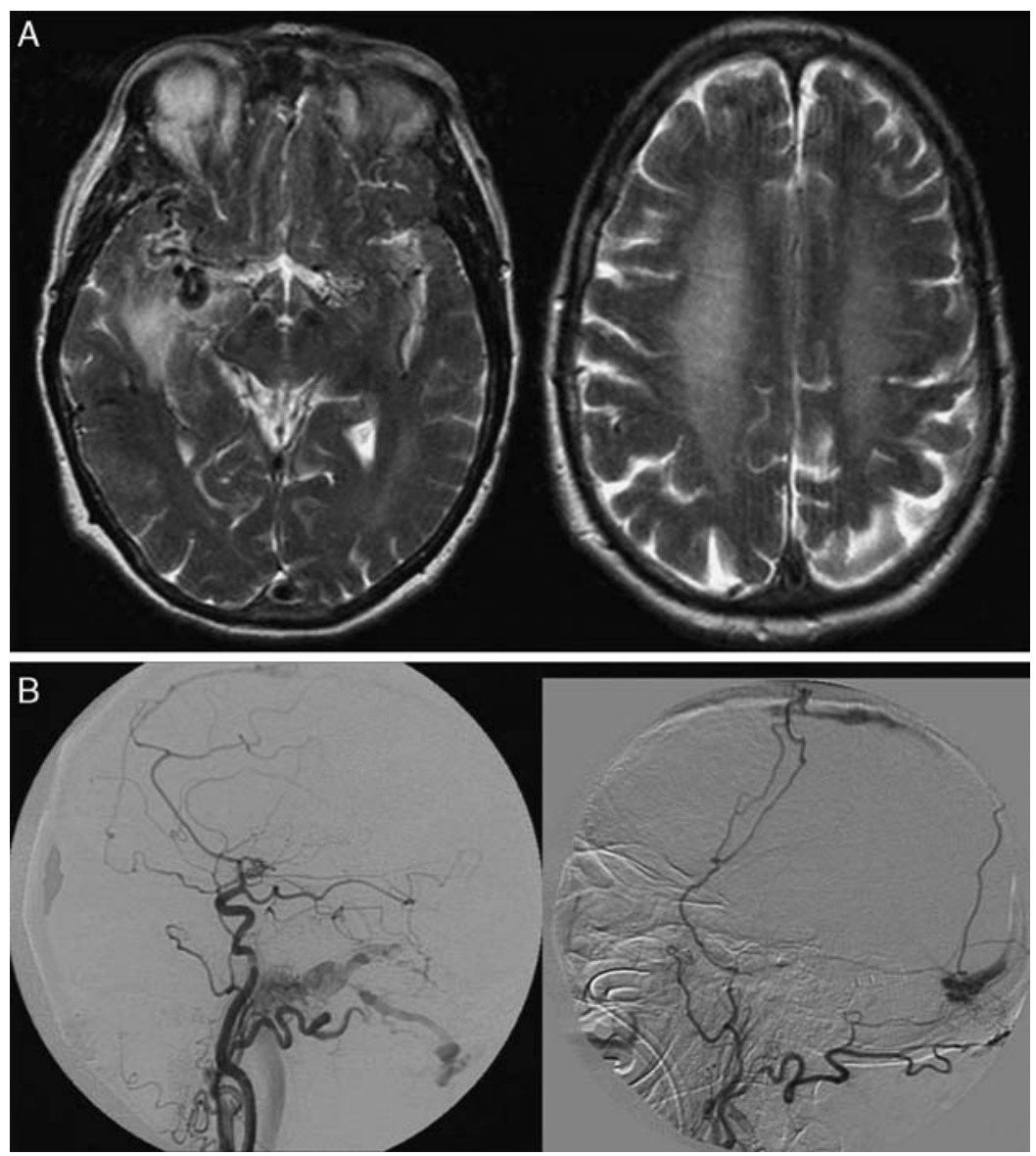

FIGURE 1. A, MRI: axial T2-weighted images shows multiple dilated vessels in the temporal regions, predominantly in the right temporal region where venous ectasia and hyperintensity of the white matter is identified. Hyperintensity of the centrum semiovale is also visible. $B$, Digital subtraction angiography: right common carotid and left external carotid injections, lateral views, shows multiple DAVFs of the superior sagittal sinus, torcula, and the lateral sinuses. DAVF indicates dural arteriovenous fistulas; MRI, magnetic resonance imaging.

extreme slowness of her responses, limitations in right hand manipulation due to pyramidal impairment, and fatigue. Meanwhile, to address the issue of a thrombophilic or inflammatory condition as the cause for the DAVFs, a serum autoimmune and anticoagulation evaluation proved negative. Also negative was the study for most relevant thrombophilic genetic polymorphism such as Factor V Leiden mutation and the GA20210 mutation of the prothrombin gene. A second endovascular treatment was performed 2 months after the first one, through the right occipital artery. It was intended to close more dural shunts, improve the clinical outcome further, and decrease the risk of brain hemorrhage. The patient did not improve after the embolization session and oral anticoagulation was started, because at this time, it was thought that the risk of additional thrombotic events outweighed the risk for serious bleeding. The patient slowly improved in the following weeks and maintained anticoagulation in an international normalized ratio range between 2 and 3 .

Three months after the first cognitive assessment, the patient was reevaluated. She was cooperative but had psychomotor slowness, some degree of inattention, and a right hand manipulation deficit that compromised the evaluation of limb praxias, writing, and right hemisphere functions like visuoconstructive abilities. The MMSE score was then 17, compatible with mild dementia and indicating some improvement as compared with the previous assessment. The patient had temporal disorientation and a verbal memory deficit in spontaneous recall but preserved recognition, indicating left temporal neocortical impairment. In addition, she had a verbal comprehension deficit but preserved naming, with altered digital agnosia and acalculia.

She was seen at 6, 9, and 12 months and had partial autonomy being able to feed herself and walk with assistance.

\section{DISCUSSION}

Intracranial DAVFs consist of direct connections between meningeal arteries and veins within the leaflets of the dura, and they present most commonly with headache, ptosis, tinnitus and less frequently with neurological deficits associated with intracranial hemorrhage. ${ }^{6}$ Particularly in older populations, DAVFs may present with a selective cognitive dysfunction like amnesia, a regional pattern such as Gerstmann syndrome, or even full-blown dementia. ${ }^{2,7-9}$ Our patient developed a rapidly progressive dementia, mimicking prion disease encephalopathy. In our view, the explanation for this particularly dramatic presentation is the huge number of DAVFs that led to global brain dysfunction through a combination of hypoperfusion of the cerebral cortex due to venous hypertension and white matter venous infarcts. ${ }^{8,10}$ Clinical improvement immediately after the first embolization indicates that venous hypertension was a critical mechanism in determining the acute profile and encephalopathic presentation. In contrast, the stable clinical improvement achieved later with anticoagulation suggests that 
progressive venous thrombosis was probably the most important and pervasive mechanism accounting for the dementia syndrome.

The mechanism underlying the formation of DAVFs is a matter of debate. It has been suggested that sinus thrombosis is related to its development, and there are several animal models showing that elevated sinus pressure after sinus thrombosis may lead to the formation of DAVFs. ${ }^{11}$

In cases with multiple complex dural fistulas, there is a strong association between DAVFs and venous sinus occlusion/stenosis, and because of this, it is especially difficult to ascertain which one is the primary event. Our case is characteristic of this debate, because there was an unequivocal demonstration of venous thrombosis and a clear clinical improvement with anticoagulation, but we could identify neither a primary condition for thrombosis nor a thrombophilic predisposition. As in the vast majority of DAVFs described in the literature, ${ }^{12}$ the results were negative for the most common and significant genetic mutations and polymorphisms associated with thrombophilia.

The treatment goal in these patients should be a complete cure by endovascular embolization and/or surgery or, in the event that goal is unattainable, relieving venous hypertension should be the target. With multiple, complex dural fistulas, as in our case, these interventions may be insufficient. Medication in this situation should consider the risk for complications, the natural course of the DAVFs, and the degree of neurological deterioration. In our patient, the endovascular procedures failed to completely treat the DAVFs, and a surgical approach was not feasible. The decision to introduce oral anticoagulation was imposed by the increasing venous thrombosis burden that was considered to outweigh the risk of a major cerebral hemorrhage. Neurologists should maintain a high degree of suspicion to avoid missing the diagnosis DAVFs that are potentially treatable lesions.

\section{REFERENCES}

1. Gonçalves MB, Maia O Jr, Correa JL, et al. Dural arteriovenous fistula presenting as thalamic dementia. Arq Neuropsiquiatr. 2008;66:264-267.

2. Hasumi T, Fukushima T, Haisa T, et al. Focal dural arteriovenous fistula (DAVF) presenting with progressive cognitive impairment including amnesia and alexia. Intern Med. 2007;46:1317-1320.

3. Weigele JB, Chaloupka JC, Lesley WS. Galenic dural arteriovenous fistula: unusual clinical presentation and successful endovascular therapy. Case report. J Neurosurg. 2002;97:467-470.

4. Folstein MF, Folstein SE, McHugh PR. "Mini-mental state". A practical method for grading the cognitive state of patients for the clinician. J Psychiatr Res. 1975;12:189-198.

5. Guerreiro MSA, Botelho MA. Brief evaluation of mental state and tests in dementia. Study Group of Aging and Dementia. 2003;27-32.

6. Cognard C, Gobin YP, Pierot L, et al. Cerebral dural arteriovenous fistulas: clinical and angiographic correlation with a revised classification of venous drainage. Radiology. 1995;194:671-680.

7. Hirono N, Yamadori A, Komiyama M. Dural arteriovenous fistula: a cause of hypoperfusion-induced intellectual impairment. Eur Neurol. 1993;33:5-8.

8. Waragai M, Takeuchi H, Fukushima T, et al. MRI and SPECT studies of dural arteriovenous fistulas presenting as pure progressive dementia with leukoencephalopathy: a cause of treatable dementia. Eur J Neurol. 2006;13:754-759.

9. Hurst RW, Bagley LJ, Galetta S, et al. Dementia resulting from dural arteriovenous fistulas: the pathologic findings of venous hypertensive encephalopathy. Am J Neuroradiol. 1998;19:1267-1273.

10. Bernstein RA, Gress DR. Dural arteriovenous fistulas and the neurology of venous hypertension. Semin Cerebrovasc Dis Stroke. 2004;4:168-175.

11. Zhu Y, Lawton MT, Du R, et al. Expression of hypoxia-inducible factor-1 and vascular endothelial growth factor in response to venous hypertension. Neurosurgery. 2006;59:687-696.

12. Gerlach R, Boehm-Weigert M, Berkefeld J, et al. Thrombophilic risk factors in patients with cranial and spinal dural arteriovenous fistulae. Neurosurgery. 2008;63:693-698. 